\title{
Improving Upper Extremity Motor Skills in Girls with Rett Syndrome Using Virtual Reality
}

\author{
Kourtney M. Mraz ${ }^{1, *}$, Gina Amadio ${ }^{1}$, Pamela Diener $^{2}$, Grace Eisenberg $^{1}$ and Jack R. Engsberg ${ }^{1}$ \\ ${ }^{1}$ Washington University in St. Louis-School of Medicine, Program in Occupational Therapy, 4444 Forest Park \\ Ave, Saint Louis, MO 63018, USA \\ ${ }^{2}$ Georgetown University-School of Medicine, Department of Neuroscience, 3970 Reservoir Rd, NW, \\ Washington, DC 20057, USA
}

\begin{abstract}
Introduction: Rett Syndrome is a genetic disorder that limits a girl's ability to use her upper extremities for daily activities, such as dressing and playing. One possible intervention to improve upper extremity function in this population is virtual reality, which can be used to increase activity demands during therapy sessions.

Objectives: To determine the feasibility of using internet-based virtual reality intervention for Rett Syndrome (RTT-IVR), to decrease hand wringing/mouthing and increase hand and arm movements away from the midline by identifying attributes and limitations to the proposed intervention.

Materials and Methods: Using FAAST Software and Microsoft Kinect sensor, RTT-IVR was trialed with 6 girls with RTT. Upper extremity movements were used to play free Internet games as means of increasing repetitions and purposeful arm movements. Data regarding attributes and limitations of the RTT-IVR intervention were collected via observation and post-session parent interviews.

Results: Interviews and observation revealed successful game play when games were motivating, clearly established cause and effect, and matched level of cognitive ability of the participant. Limitations include technological glitches regarding Kinect sensor sensitivity and identifying appropriate games for each participant's interests and abilities.

Conclusion: Internet based virtual reality interventions for girls with RTT should be highly individualized to increase motivation and success of intervention.
\end{abstract}

Keywords: Rett Syndrome, internet-based virtual reality, Microsoft Kinect, upper extremity movements; qualitative analysis.

\section{INTRODUCTION}

Rett Syndrome (RTT) is an X chromosome-linked genetic disorder almost exclusively seen in females. RTT results in orthopedic and neurologic dysfunctions such as scoliosis, ataxia, apraxia, intellectual disability, loss of acquired speech, and decreased use of hands in everyday activities [1]. Stereotypical behaviors of RTT include bilateral hand wringing and mouthing movements at midline which precludes the individual from using their upper extremities in purposeful tasks such as homework, playing, communication, and other activities of daily living [1]. The probable cause behind the neurological effects of RTT (i.e. motor deficits) is a mutation in the gene that encodes for methyl-CpG protein 2 (MeCP2). MeCP2 plays a role in the development of mature neurons; when development of mature neurons is inhibited, gait and motor abnormalities can occur. MeCP2 deficits can also lead to changes in cortical excitation and inhibition, which has been associated with the stereotypical repetitive hand wringing/mouthing movements seen in individuals

*Address correspondence to this author at 5837 Nina Place, St. Louis, MO 63112, USA; Tel: 815-450-0069; Fax: 314-286-1601; E-mail: kmraz@wustl.edu with RTT [2]. Current treatment for RTT includes a myriad of interventions including physical, occupational, hippo-, aquatic, and music therapies aiming to minimize decline in functional arm movements or promote improvement in skilled function (i.e. feeding and play skills). Eye gaze technology is commonly used by girls with RTT to promote communication; and research continues to investigate use of eye gaze to assess cognition, communication, and visual processing in this population [3-6]. Evidence to support the efficacy or proper dosing of these interventions individually or in combination is limited at best. Consequently, best practice for treatment remains unknown $[1,7]$.

For skill acquisition to take place, multiple motor learning principles are used to guide current therapy intervention. Increasing the number of times a skill is practiced is one of the more effective principles for improving performance within a session [8, 9]. Although a definitive number of repetitions needed for optimal motor learning is still unknown, current research suggests that 300 or more movement repetitions is (1) feasible within a 1-hour therapy session and (2) linked to better motor outcomes [10]. However, in reality, only 
30-40 functional upper extremity movement repetitions are being completed in a traditional therapy session lasting on average $36 \pm 14$ minutes [11]. The discrepancy in number of motor repetitions suggests room for improvement in therapeutic motor training practices to improve skill attainment. Virtual Reality (VR) is a highly motivating intervention $[12,13]$ that can promote 300-600 movement repetitions in one 40minute session [14, 15], suggesting that VR intervention creates an opportunity to meet the number of repetitions suggested for optimal lasting neural changes related to learning of motor skills. VR enables ample practice and development of new movement patterns that can be used in everyday activities. VR training has been successful in increasing repetitions, motor function, movement kinematics, quality of life, and participation in children with cerebral palsy and adults with chronic stroke [16-20].

The use of VR in rehabilitation can create a wide range of interactive environments and incorporate motor learning principles to increase activity demands in a single therapy session. VR intervention should therefore be directed towards motor training and customized for the individual. Addressing deficits such as upper extremity disuse associated with RTT may lead to motor improvements beyond the virtual environment. The purpose of this investigation was to determine the feasibility of using internet-based virtual reality intervention for RTT, to decrease hand wringing/mouthing and increase hand and arm movements away from the midline.

\section{METHODS}

\section{Subjects}

Six participants were recruited through a neurologist at St. Louis Children's Hospital, Missouri. All participants lived in the Saint Louis, Missouri metropolitan or surrounding areas. Inclusion criteria for participation was (1) diagnosis of RTT, (2) no functional limitations of upper extremity passive range of motion, and (3) ability to understand cause and effect as reported by parent or caregiver. Informed consent was obtained by a parent/guardian of the potential participant at the beginning of the participant's first session. The study was approved by the Washington University Medical Institutional Review Board.

\section{Device Description}

The Microsoft Kinect sensor, Flexible Action and Articulated Skeleton Toolkit (FAAST) software, and internet games were used to create the VR intervention [14]. Due for the need for easy access to virtual environments and the variety of interests of the participants, free internet games were used for participant-specific VR intervention. Freeing one from having to touch a controller, the Kinect [21] captures upper extremity movement in order to control the internet virtual reality (IVR) games. The upper extremity movements that control game play are configured by the therapist through the FAAST software [14, 22]. For example, using FAAST, the therapist defines a specific threshold for right shoulder flexion to activate the jump action in a selected game. The participant then attempts the defined movement while playing the game. The Kinect registers the movement and if the defined movement threshold is met, the jump function will activate, resulting in successful game play.

\section{Intervention}

Each participant completed a 1 to 2 hour session to trial the internet-based virtual reality intervention for Rett Syndrome, or RTT-IVR. During the session, the intervention was described to the participant and attending parents. Parents were given the opportunity to try the intervention before their daughter in order to solidify understanding of what their daughters were being asked to do. The RTT participants then attempted 3-5 pre-selected games of varying cognitive difficulty, trialing different movement thresholds and body positions throughout. Specifically, the participant was positioned in front of the Kinect sensor and large television screen where the selected game was displayed for viewing. The participants were either asked to separate their hands or reach forward (elbow extension) to participate in game play. Each correctly performed movement resulted in a successful gameplay action. Verbal encouragement was provided and movement thresholds were adjusted in FAAST to promote as much success as possible. The sessions concluded with a feedback session with parents. Parents were asked what they liked about the intervention, what they thought could be improved, and what games they thought were most motivating for their daughter. If possible, the participant was asked which game they liked best using their preferred communication device.

Information regarding attributes and limitations affecting success of the intervention for each participant was used to adjust the session for the following participant. The following figure describes the plan for determining feasibility of the RTT-IVR intervention across sessions. 


\begin{tabular}{|c|c|c|c|}
\hline Phone Interview & Feasibility Session & Modification & Repeat \\
\hline Gathered & Trial RTT-IVR with & Made changes to & Three step process \\
\hline demographic & participant. & intervention & repeated with new \\
\hline information and & Conducted parent & according to parent & participant using \\
\hline $\begin{array}{l}\text { interests for all } \\
\text { participants. }\end{array}$ & feedback interview. & $\begin{array}{l}\text { feedback and session } \\
\text { observation. }\end{array}$ & modified approach. \\
\hline
\end{tabular}

Figure 1: Methodology.

\section{Data Collection}

To inform the feasibility of the RTT-IVR intervention, the primary outcome of each session was to determine attributes and limitations of the intervention. Due to the descriptive nature of this study, qualitative data were collected via investigator observation and post-session parent interviews. Observation notes were made during and after each participant session regarding participant reaction and adjustments made to the intervention to promote participants' success. Notes of two investigators were compared following each session. Parent interviews following each data session were recorded via audio recorder.

\section{Data Analysis}

Recorded parent interviews were transcribed by the primary investigator. Transcriptions were reviewed for accuracy by a second investigator. Using content analysis techniques [23, 24], transcriptions were first reviewed for units, then organized into categories, and finally reviewed for relationships between themes. Units consisted of words, phrases, or sentences that represented a concept. Units were then grouped into categories related to improvement of the trialed intervention. To ensure quality of analysis, two investigators performed individual content analyses of the same text and then compared themes and categories derived from the transcribed text. Discrepancies were discussed and converged into appropriate categories.

\section{RESULTS}

\section{Subjects}

Participant 1 was a 16-year-old female with RTT. She was nonverbal and participated in typical education classrooms with the assistance of a personal aide. She used a dependent manual wheelchair for mobility, and was able to ambulate with physical assistance. She would often wring her hands at midline throughout the day but might occasionally grab a spoon, tap a communication switch at school, rub her eyes or hair, or pat something (described as an act of endearment). Participant 1 was reported to have occasional seizures. Her interests included music, food, and social interaction. Participant 1 was seen in the Human Performance Laboratory at Washington University in St. Louis.

Participant 1 demonstrated ability to extend her elbow for the reaching movement needed to play internet games, but required hand-over-hand assistance to complete the movement in conjunction with the game. The participant demonstrated ability to initiate the wanted movement. She repeatedly performed small movements without attending to the game screen, suggesting that an understanding of cause and effect for this task was not fully established. Throughout this participant's session, investigators troubleshot many aspects of the session including: how to provide hand-over-hand assistance without interfering with the Kinect sensor, how best to position the participant (e.g. wheelchair versus computer chair with arms), and how to motivate the participant beyond the game (e.g. enthusiasm, holding an object, and reaching for an object). Best solutions for these challenges were not identified within the session. Data from the post session parent interview suggested that the gaming experience should be "motivating" and "age appropriate". Upon interview, the participant's personal assistant also stated that 1-2 of games trialed may have been too visually stimulating, saying "I wonder if she sees those movements on the screen" and "it 
might make her eyes tired". The mother also stated "once the [technology] is fine-tuned, she will have more cause and effect [understanding]....and could go longer periods of time without getting frustrated or tired". As a result, we focused on troubleshooting technological glitches during game play and made no changes to the game selection to be trialed by Participant 2 .

Participant 2 was a 21-year-old female with RTT. She was nonverbal and used both her eye gaze and her hands with picture boards for communication and decision making. She demonstrated hand wringing tendencies. She used a dependent stroller for mobility and could ambulate with physical assistance. Participant 2 was reported to have seizures, beginning approximately at the age of 6 , however they were controlled by medication. Her interests included movies and music. Participant 2 was seen in the Human Performance Laboratory at Washington University in St. Louis.

Participant 2 required hand-over-hand assistance to perform the defined movements for successful game play. Throughout the session she showed no progress towards performing the movements independently. Although her eye gaze was intensely focused towards the game screen, it is unclear if she understood cause and effect related the task. Data from the post session parent interview suggested that providing the participant a choice between games using her established communication preference may promote motivation. Additionally, the participant's father agreed that simplistic games that were not too overwhelming visually, were best. Finally, the father suggested that music, such as Disney songs, may be both relaxing and motivating for his daughter.

As a result of information gathered from participant 1 and 2, we made the following changes: We found simpler, single switch/input, games with less cognitive requirements and larger, clearer visuals. For example, instead of a game with a small character where the participant was responsible for making the character "jump" at the appropriate time for game success, we found a game where upon activating a single switch, a rocket with a plain background exploded into large fireworks on the screen. Ultimately, our goal was to find simple, clear games that would better promote establishing cause and effect.

Participant 3 was a 10-year-old female with RTT. She was nonverbal and participated in a typical $4^{\text {th }}$ grade classroom at school. She made decisions through presentation of choices and selected with eye gaze. She ambulated independently in her home without use of mobility device. Hand wringing behavior was present, as well as rhythmic body rocking. Her parents reported no seizure activity. She enjoyed music and beauty products. Participant 3 was seen in her home.

Participant 3 demonstrated the ability to separate her hands away from midline independently resulting in successful game play. Participant 3 attended to the game screen for the majority of the session, demonstrated by directing eyes toward the screen and actively participating in game play. Investigators and parents provided verbal encouragement and reward for movement attempts. It was clear that she understood cause and effect, yet unclear whether she was more motivated by game success or response from parents/investigators. Data from the post session parent interview suggested that games should be "age appropriate", "provide choice to the participant", and be related to [the participants'] interests, stating "some girls might like this [interest], but some girls may not". Music was also stated to be "the biggest key" factor for game choice. Additionally, the parents suggested that the player should always be rewarded no matter what movement is performed, stating "whatever motions they do, [the software] should take any motion, and [the game] needs to do an action". The parents also suggested that games should provide opportunity for grading difficulty for challenging cause and effect by using multi-input controls so "this movement results in [this game] action, and that movement results in [a different game] action."

As a result we made the following changes: In order to increase success, we increased time spent training participant in cause and effect. We did so by allowing the participant to control the playing of music or favorite video by performing the wanted arm movements prior to game play. In order to provide immediate reinforcement for performing the wanted action, we chose not to use the FAAST or Kinect sensor for this step, due to inconsistency and the need to provide hand over hand assistance, and instead manually activated the music/video from the keyboard.

Participant 4 was a 35-year-old female with RTT. She had retained a few verbal phrases for communication. She used a dependent wheelchair for mobility, could stand with assistance, and assist with repositioning in wheelchair. Her stereotypic hand movements included hand to hair and mouth 
movements, as well as rhythmic body rocking. She was fed via a gastrointestinal tube due to her no food by mouth status. She has had seizures since the age of 4 , however currently takes medication to control them. Her interests included all types of movies, Disney music, and using her stander at home. Participant 4 was seen in the Human Performance Laboratory at Washington University in St. Louis for one 2-hour session due to implementation of new cause and effect training techniques described below.

Participant 4 required hand-over-hand assistance for completion of wanted movements. Reaching and hand separation was attempted. Participant demonstrated ability to initiate upper extremity movement when requested, but not in response to the game. Shortly after game play was unsuccessful, the remainder of the session was focused on establishing cause and effect using hand-over-hand assistance and the investigator controlling the game in response to any movement made by the participant to promote connection between the two components. Cause and effect was also practiced by allowing the participant to control the 'play' button on a video. For example, once the video was paused, the participant controlled when the video would play by initiating the wanted movement, while the investigator would assist in completion of the movement. Understanding of cause and effect was present, however more time would be needed to fully connect cause and effect between movements and game play. Data from the post session parent interview suggested that the most important component for success was that games should be related to participant interests, for example, Disney Channel television shows, animals, and music videos. It was suggested that games have human characters as opposed to animated ones. As a result, we would continue to use music and videos for initial cause and effect training before beginning game play. To address technical issues, the Microsoft Kinect V2 sensor was used in place of the original Kinect sensor.

Participant 5 was an 8-year-old female with RTT. She attended school in special education classes. She has used a few verbal phrases and a keyboard based "talker" device for communication. She ambulated independently. She used her hands to activate her communication device, play, eat, and don jackets. Her stereotypic hand movements included twirling her hair and pinching her fingers together in a tripod pinch pattern. She had no documented seizure history, but took anti-seizure medication due to seizure precursor activity. Her interests included animals, Disney princesses, music, and Star Wars. Participant 5 was seen in the Human Performance Laboratory at Washington University in St. Louis.

Participant 5 was independent in performing reaching movements with both arms and standing during game play. She clearly had understanding of cause and effect based on performance in initial cause and effect training, which carried over into successful game play. Important to note, is that Participant 5, compared to the previous 4 participants, displayed the most obvious interest in the selected games, frequently nodding her head and clapping when asked if she would like to play again. Additionally, due to the nature of school related computer games described by her mother, it was possible that Participant 5's cognitive and physical capacities suggest possibility for more challenging games.

Data from parent interview for Participant 5 was mostly gathered during the session due to unique challenges Participant 5 presented to the intervention. For instance, we identified that more challenging games may be more appropriate for this participant. Her mother suggested games similar to ones she played in school where she would click and drag letters into two separate bins on a touch screen. When the mother was asked if she thought this intervention was something she could see her daughter doing regularly in her home, she stated that she thought it was possible for her daughter, "especially because she has not hit her regression stage, and we are trying to challenge her as much as we can so the outcomes of her eventual regression might be better". Beyond our troubleshooting conversations throughout the session, Participant 5's mother did not have any additional opinions on how to improve the RTT-IVR intervention.

Participant 6 was a 10-year-old female with RTT. She was nonverbal, but communicated using eye gaze and a switch. She used a dependent manual wheelchair for mobility, and was able to ambulate with physical assistance. Her stereotypic hand movements included hand to hair and mouth movements, as well as picking at her skin. Participant 6 was reported to have occasional seizures, and she takes medication to control them. She also had scoliosis. Her interests included music, Disney Princesses, Mickey Mouse, and Taylor Swift. Participant 6 was seen in the Human Performance Laboratory at Washington University in St. Louis for one 1-hour session.

Participant 6 demonstrated the ability to separate her hands away from midline independently, however, 


\begin{tabular}{|c|c|c|c|c|c|c|}
\hline Participant & Age & Hand Stereotype & $\begin{array}{c}\text { Upper Extremity } \\
\text { Use }\end{array}$ & $\begin{array}{l}\text { Education } \\
\text { Status }\end{array}$ & Comm. Preference & $\begin{array}{l}\text { History of } \\
\text { Seizures }\end{array}$ \\
\hline 1 & 16 & Wringing & Comm. Switches & $\begin{array}{c}\text { Typical } \\
\text { classroom; } 1 \text { on } 1 \\
\text { aide }\end{array}$ & Comm. Devices & Yes \\
\hline 2 & 21 & Wringing & Comm. Boards & Unknown & $\begin{array}{l}\text { Picture boards, eye } \\
\text { gaze }\end{array}$ & Yes \\
\hline 4 & 35 & $\begin{array}{l}\text { Mouthing; hands to } \\
\text { hair }\end{array}$ & Grasp objects & Unknown & $\begin{array}{c}\text { Verbal phrases, eye } \\
\text { gaze }\end{array}$ & Yes \\
\hline 5 & 8 & $\begin{array}{l}\text { Hands to hair; } \\
\text { pinching }\end{array}$ & $\begin{array}{l}\text { Pointing, comm. } \\
\text { Device }\end{array}$ & $\begin{array}{l}\text { Special education } \\
\text { class room }\end{array}$ & $\begin{array}{l}\text { Comm. Device; } \\
\text { some vocalization }\end{array}$ & $\begin{array}{l}\text { Pre-cursor } \\
\text { activity }\end{array}$ \\
\hline
\end{tabular}

Figure 2: Participant characteristics.

\begin{tabular}{|c|}
\hline $\begin{array}{l}\text { Participants } 1 \text { and } 2 \\
\text { Trialed single input games that were identified as too cognitively complex and time intensive. Trialed various } \\
\text { seating (e.g. wheelchair, computer chair, desk chair) to allow for hand-over-hand assistance with least } \\
\text { interference for Kinect sensor. }\end{array}$ \\
\hline $\begin{array}{l}\text { Participant } 3 \\
\text { Simplified all games to single input, cause and effect ga } \\
\text { home, instead of the laboratory, while standing indepen } \\
\text { previous session }\end{array}$ \\
\hline $\begin{array}{l}\text { Participant } 4 \\
\text { Established cause/effect after initial trial of games usin } \\
\text { game play; emphasized proper positioning for stability }\end{array}$ \\
\hline $\begin{array}{l}\text { Participant } 5 \\
\text { Established cause/effect prior to game play using Participant } 4 \text { methods, used the updated Microsoft Kinect V2 } \\
\text { sensor to alleviate sensor glitches. }\end{array}$ \\
\hline $\begin{array}{l}\text { Participant } 6 \\
\text { Established cause/effect prior to game play using Participant } 4 \text { and } 5 \text { methods, used the updated Microsoft } \\
\text { Kinect V2 sensor to alleviate sensor glitches, although not very successful. }\end{array}$ \\
\hline
\end{tabular}

Figure 3: Modifications to RTT-IVR intervention sessions from participant to participant.

her wheelchair was fixed with a large headrest to support her neck which impeded the Kinect's ability to detect her. She was transferred to a chair, but her small stature prevented her shoulders' from being elevated above the back of the chair. As a result, she required hand-over-hand assistance and support in order to been seen by the Kinect. She completed a forward reach with hand-over-hand assistance. Participant 6 attended to the game screen for the majority of the session, as demonstrated by maintaining eye gaze on the screen. Investigators and mother provided verbal encouragement and tried to motivate her with a ball. It was unclear if she understood cause and effect, which may have been due to the Kinect's inconsistency in detecting her. Cause and effect was practiced by allowing the participant to control the 'play' button on a video. Data from the post session parent interview suggested that games should "include more real life characters". Additionally, music was suggested to be a motivator. When asked what goals she had for her daughter, Participant 6's mother stated that she "hasn't given it much thought", but she mentioned that her daughter is unable to use her hands to feed herself. Her mother reported that her daughter would be more motivated to touch tangible object such as an iPad screen. 


\begin{tabular}{|c|c|c|c|c|c|c|}
\hline Participant & Position & $\begin{array}{l}\text { Movement } \\
\text { performed }\end{array}$ & Assist level & $\begin{array}{l}\text { Initiation of } \\
\text { movement }\end{array}$ & $\begin{array}{l}\text { Established } \\
\text { Cause/ Effect }\end{array}$ & $\begin{array}{c}\text { Response / } \\
\text { Game Success }\end{array}$ \\
\hline 1 & Seated & Unilateral Reaching & Hand-over-hand & Yes & No & Low \\
\hline 2 & Seated & Unilateral Reaching & Hand-over-hand & No & No & Low \\
\hline 3 & Standing & Hand separation & Independent & Yes & Yes & High \\
\hline 4 & Seated & $\begin{array}{l}\text { Hand separation, } \\
\text { reaching }\end{array}$ & Hand-over- hand & Yes & Yes & Moderate \\
\hline 5 & $\begin{array}{l}\text { Standing\& } \\
\text { Seated }\end{array}$ & Reaching & Independent & Yes & Yes & High \\
\hline 6 & Seated & Reaching & Hand-over-hand & Some & Yes & Low \\
\hline
\end{tabular}

Figure 4: Summary of session details, participant response, and general outcomes.

\section{DISCUSSION}

The body of knowledge regarding the feasibility of virtual reality interventions for individuals with RTT is limited at best. Therefore, the purpose of this investigation was to determine the feasibility of using an internet-based VR intervention for individuals with RTT to decrease hand wringing/mouthing and increase hand and arm movements away from the midline.

Limitations of this study included a small sample size for trialing the RTT-IVR intervention. Additionally, technological glitches hindered successful, participant activated game play. For instance, tracking capabilities of the Microsoft Kinect sensor resulted in occasional tracking of the chair or wheelchair instead of the upper extremity, especially when the hands were clasped at midline, and delayed response time for successful game play. In the final feasibility session, the updated Kinect V2 sensor was used because the sensor has improved tracking capabilities and response time during game play. Another limitation of the Microsoft Kinect sensor is that if more than one skeleton (e.g. a parent or therapist standing near the participant) was visible in the sensors field of view, the software was no longer able to register the participant's movements to play the game successfully. This was most troublesome in three participant scenarios where handover-hand assistance was necessary for establishing the goal movement and cause and effect. The research team problem solved this issue by having one researcher provide assistance to the participant while another researcher controlled the game as the desired movements occurred. Limitations in data analysis included not having a pre-determined list of discussion questions (e.g. an exit interview) for parents following each participant's session. Instead, parent interviews consisted of comments about what they observed their child doing and what factors they thought would improve their child's experience and success in game play. There was a need for more objective measures of participant progress, engagement, and enjoyment related to their intervention experience. Although one attribute to this intervention included having access to seemingly endless numbers of free internet games, investigators had difficulty finding games appropriate for the RTT population. The biggest difficulty was finding games that were collectively simple (e.g. single input), visually stimulating, motivating, and age appropriate. It is possible that by expanding the internet game bank to include games under $\$ 10$, access to RTT appropriate games may increase significantly. After having Participants 4 and 5 practice cause and effect using the Kinect and FAAST system to start and stop music videos, it became apparent that the current methodology could be used in many ways for girls with RTT by not limiting intervention to online games alone, but venturing into any task that can be performed virtually.

The three categories that emerged from the interview data were (1) motivators of girls with RTT that may influence interest in games, (2) participant factors influencing participation and success in VR intervention, and (3) game factors influencing successful VR intervention. Units from these categories inform needs for appropriate and successful VR intervention for girls with RTT.

\section{Identified Motivators for Successful Game Play}

Units extracted from parent interview data relating to participants motivation include "music", "videos", "Disney", "food", and "siblings/friends". Music was found to be the most frequently identified motivator, appearing in all 6 parent interviews. Parent's believed that games should have enjoyable background music to maintain interest and promote relaxation of the girl playing the game. Music, used as a means or part of therapeutic intervention protocols, has been found to 
be enjoyable and motivating for girls with RTT, sometimes promoting voluntary and purposeful hand use [25, 26, 27, 28]. Additionally, parents suggested that games incorporate participant control of music. For instance, a game where music plays while the participant is performing the goal arm movement (i.e. hands separated away from midline) and music stops when the goal movement is stopped (i.e. hands return together at midline). Food as a motivator was mentioned in two parent interviews as a way to reward participation in game play. Videos of Disney movies and television shows were identified as interests and motivators for 5 of the 6 participants, suggesting that online games related to these topics may provide additional motivation for game play. Two parent interviews identified social interaction as a goal for the IVR-RTT intervention, mentioning that participants being able to play these games with friends and siblings in the home would be ideal.

One parent suggested that "real characters" would motivate her daughter to engage in game play. She also explained that her daughter would be more likely to reach for a tangible object as opposed to moving to operate the Kinect. For example, her daughter may interact with a human or reach for a switch before she would comprehend the connection between her movement and activation of the Kinect.

\section{Participant Factors Influencing Participation and Success in VR Intervention}

Parent interviews after the first three sessions suggested a need for participants to better establish cause and effect for successful game play. For example, one parent suggested more simple games where "an object moves when [participant] makes a movement" while another parent stated "with a little more cause and effect, I think she will enjoy the game more". Therefore, participants 4,5 , and 6 received more rigorous practice in establishing cause and effect using single switch video and music operations prior to attempting games. The need for cognitive training in cause and effect is supported by the variable, but characteristic decline in cognitive abilities in girls with RTT following stages of regression [29, 30]. Two parent interviews mentioned that arm movement thresholds should be chosen based on participants' already established natural movements to increase likelihood that the participant will achieve the goal movement and have increased success in game play. Additionally, all six parent interviews mentioned providing the participant the opportunity to choose which game they would like to play using their preferred method of communication, congruent with contemporary practices of providing client-centered occupational therapy [31, 32]. Parents linked these factors to increased motivation and success for their daughters.

Participant 6 was unable to successfully operate the Kinect due to the size of her wheelchair; the headrest prevented the sensor from detecting her skeleton. Additionally, her repetitive hand movements included mouthing and hair pulling, which made is difficult to use hands to midline to deactivate the game as this was not a movement that she frequently engaged in.

\section{Game Factors Influencing Successful VR Intervention}

Units derived from the data that referred to game specific factors included "age appropriate", "simple games", "clear visuals and sounds" and "fine-tuning" the technology. Three parent interviews mentioned age appropriateness as a critical aspect for game selection. Complementing the identified need for establishing cause and effect with participants, parents suggested simple games to help establish the connection that performance of the goal movement is what causes the reaction on the screen. While simple games were identified as beneficial by all parents, Participant 3's parents suggested interest in games with multiple inputs so participants had more control in the game as well as an opportunity for increasing game difficulty. For Participants 3 and 5 who were both younger, educational games incorporating letters and colors were identified as a way of increasing cognitive challenge. Additionally, one game trialed with 5 of the 6 participants was visually complex, or busy, possibly resulting in tiring participants' eyes and limiting the participants' ability to visually identify what affect their performed movement had on the game. This issue also prompted parents to suggest games with clear visuals and auditory sounds to assist participants in following game play. In addition to the games themselves, two parent interviews identified flaws with the Kinect sensor and FAAST system (e.g. displaced markers and delayed inputs) which limited the participant's success in game play. One parent suggested that all arm movements attempted by the participant should be rewarded with a reaction on the game screen; this requires that the technology be accurate in tracking movements and appropriate movement thresholds defined for high success. Another parent stated that fine-tuning these flaws may make game play more enjoyable and less frustrating for the participant, while 
helping to establish cause and effect. Refining technological glitches and structuring games specifically to produce player success promotes errorless, or error-reduced, learning - a method of motor learning shown to improve learning of motor skills in children with intellectual disability [33]. Finally, phrases like "what one girl may like, another may not" suggests a need for client centeredness and a large game library from which the participant may choose.

\section{Attributes to the RTT-IVR Intervention}

Attributes to the RTT-IVR intervention were identified primarily through clinical judgment and observation. Some positive aspects of the intervention were supported by comments made by parents during interviews. The following attributes were identified: (1) vast availability of free internet games; (2) software for intervention is inexpensive for home or clinic use; (3) software provides a large range for thresholds, movement definitions, and number of inputs for game play, providing multiple options for grading difficulty for participant experience; (4) changing, or grading, movement thresholds and definitions is quick if therapist is familiar with software; (5) use of large high definition television allows for clear, visually stimulating game play experience; and (6) intervention provides interactive physical activity for RTT population.

Utilizing analysis of parent interview data and clinical observation during feasibility sessions, the following findings can be used to inform future success of the RTT- IVR intervention: (1) incorporate clientcenteredness by using participants' interests and providing choice in age-appropriate game options, (2) incorporate goal for establishing cause and effect by using single switch, visually clear games and external rewards if necessary, and (3) fine-tune glitches in technology to ensure constant reward and understanding of cause and effect through game success with reduced risk of participant frustration.

\section{CONCLUSIONS}

Findings from this study agree with existing RTT research regarding the variability of physical and intellectual characteristics amongst individuals with RTT. The variability in function across the current participants with RTT suggest the need for highly individualized approach to the RTT-IVR intervention when selecting games and movement thresholds. However, parent-identified needs for intervention success were similar across all participants, informing the possibility for a successful and generalizable intervention protocol.

\section{CLINICAL SIGNIFICANCE}

Clinically, information derived from this study supports previous knowledge of characteristics and functional abilities of girls with RTT. Based on observation, participants who responded positively to the intervention tended to be able to clearly establish cause and effect during the task, younger, and able to initiate the upper extremity movement pattern independently. The findings inform guidelines for a RTT specific internet based virtual reality intervention protocol.

\section{FUTURE WORK}

Further research is needed to investigate prolonged outcomes of internet based virtual reality intervention for girls with RTT. Therefore, if possible, the current study's participants could be invited back for 3 followup visits to eliminate short comings in the currently proposed protocol. In conjunction with additional sessions, objective outcomes of progress, functional hand use, and engagement can be measured. Feasibility and efficacy of the RTT-IVR intervention should be investigated for home and clinical use similar to what is currently being done with stroke populations [14]. Research can also look into using tasks other than online games to promote upper extremity movements using the Kinect and FAAST system. Additionally, future research may also focus on body positioning to facilitate upper extremity movements [27] and the motivating role of music during therapeutic intervention.

\section{REFERENCES}

[1] Lotan M. Rett syndrome. Guidelines for individual intervention. Sci World J 2006; 6: 1504-16. http://dx.doi.org/10.1100/tsw.2006.252

[2] Cuddapah VA, Nwaobi SE, Percy AK, Olsen ML. MECP2 in the regulation of neural activity: Rett syndrome pathophysiological perspectives. Degener Neurol Neuromuscul Dis 2015; 5: 103-16.

[3] Baptista PM, Mercadante MT, Macedo EC, Schwartzman JS. Cognitive performance in Rett syndrome girls: A pilot study using eyetracking technology. J Intellectual Disabil Res 2006; 50: $662-6$

http://dx.doi.org/10.1111/j.1365-2788.2006.00818.x

[4] Djukic A, Valicenti McDermott M, Mavrommatis K, Martins CL. Rett syndrome: Basic features of visual processing-a pilot study of eye-tracking. Pediatr Neurol 2012; 47: 25-9. http://dx.doi.org/10.1016/j.pediatrneurol.2012.04.009

[5] Rose SA, Djukic A, Jankowski JJ, Feldman JF, Fishman I, Valicenti-Mcdermott M. Rett syndrome: An eye-tracking study of attention and recognition memory. Dev Med Child Neurol 2013; 55: 364-71. http://dx.doi.org/10.1111/dmcn.12085 
[6] Sigafoos J, Kagohara D, Van Der Meer L, Green VA, O'Reilly $\mathrm{MF}$, Lancioni GE, et al. Communication assessment for individuals with Rett syndrome: A systematic review. Res Autism Spectrum Disorders 2011; 5: 692-700. http://dx.doi.org/10.1016/j.rasd.2010.10.006

[7] Lotan M, Hanks S. Physical therapy intervention for individuals with Rett syndrome. Sci World J 2006; 6: 131438.

http://dx.doi.org/10.1100/tsw.2006.187

[8] Kitago T, Krakauer JW. Motor learning principles for neurorehabilitation. Handbook Clin Neurol 2013; 110: 93103.

http://dx.doi.org/10.1016/B978-0-444-52901-5.00008-3

[9] Korman M, Raz N, Flash T, Karni A. Multiple shifts in the representation of a motor sequence during the acquisition of skilled performance. Proc Nati Acad Sci USA 2003; 100: 12492-7. http://dx.doi.org/10.1073/pnas.2035019100

[10] Birkenmeier RL, Prager EM, Lang CE. Translating animal doses of task-specific training to people with chronic stroke in 1-hour therapy sessions: A proof-of-concept study. Neurorehabil Neural Repair 2010; 24: 620-35. http://dx.doi.org/10.1177/1545968310361957

[11] Lang CE, MacDonald JR, Reisman DS, Boyd L, Jacobson Kimberley T, Schindler-Ivens SM, et al. Observation of amounts of movement practice provided during stroke rehabilitation. Arch Phys Med Rehabil 2009; 90: 1692-8. http://dx.doi.org/10.1016/j.apmr.2009.04.005

[12] Jack D, Boian R, Merians AS, Tremaine M, Burdea GC, Adamovich SV et al. Virtual reality-enhanced stroke rehabilitation. IEEE Transactions 2001; 9: 308-18. http://dx.doi.org/10.1109/7333.948460

[13] Lin J, Kelleher CL, Engsberg JR. Developing home-based virtual reality therapy interventions. Games for Health Journal 2013; 2: 34-8.

http://dx.doi.org/10.1089/g4h.2012.0033

[14] Lauterbach SA, Foreman MH, Engsberg JR. Computer games as therapy for persons with stroke. Games for Health 2013; 2: 24-8.

http://dx.doi.org/10.1089/g4h.2012.0032

[15] Laver KE, George S, Thomas S, Deutsch JE, Crotty M, Ke L, et al. Virtual reality for stroke rehabilitation. Stroke 2012; 42: 20-1.

http://dx.doi.org/10.1161/STROKEAHA.111.642439

[16] Kondo M, Gray LJ, Pelka GJ, Christodoulou J, Tam PPL, Hannan AJ. Environmental enrichment ameliorates a motor coordination deficit in a mouse model. European Journal of Neuroscience 2008; 27: 3342-50. http://dx.doi.org/10.1111/j.1460-9568.2008.06305.x

[17] Liamputtong P. Qualitative data analysis: conceptual and practical considerations. Health Promot J Austr 2009; 20(2):133-9.

[18] Nudo RJ, Plautz EJ, Frost SB. Role of adaptive plasticity in recovery of function after damage to motor cortex. Muscle Nerve 2001; 24: 1000-19.

http://dx.doi.org/10.1002/mus.1104

[19] Proffitt RM, Alanjus G, Kelleher CL, Engsberg JR. Use of computer games as an Intervention for stroke. Top Stroke Rehabil 2011; 18(4): 417-27.

http://dx.doi.org/10.1310/tsr1804-417
[20] Steenbeek D, Ketelaar M, Galama K, Gorter JW. Goal attainment scaling in paediatric rehabilitation: a critical review of the literature. Develop Med \& Child Neuro 2007; 49: 5506.

http://dx.doi.org/10.1111/j.1469-8749.2007.00550.x

[21] Microsoft News Center. New Xbox 360, Kinect sensor and "Kinect Adventures"-get all your controller-free entertainment in one complete package. July 20, 2010. www.microsoft $\quad$.com/en-us/news/press/2010/jul10/0720kinectpackagepr.aspx (accessed May 1, 2013).

[22] Suma E, Lange B, Rizzo S, Krum D, Bolas M. (2013) Work Flexible Action and Articulated Skeleton Toolkit (FAAST). http://projects.ict.usc.edu/mxr/faast/

[23] Hanson JL, Balmer DF, Giardino AP. Qualitative research methods for medical educators. Acad Pediatr 2011; 11: 37586.

http://dx.doi.org/10.1016/j.acap.2011.05.001

[24] Zhang Y, Wildemuth BM. Qualitative analysis of content. Applications of Social Research Methods to Questions in Information and Library Science 2009; 308-19.

[25] Kerr AM, Montague J, Stephenson JB. The hands, and the mind, pre- and post-regression, in Rett syndrome. Brain Dev 1987; 9: 487-90.

http://dx.doi.org/10.1016/S0387-7604(87)80070-0

[26] Lotan M, Isakov E, Merrick J. Improving functional skills and physical fitness in children with Rett syndrome. J Intellectual Disabil Res 2004; 48: 730-5. http://dx.doi.org/10.1111/j.1365-2788.2003.00589.x

[27] Wigram T, Lawrence M. Music therapy as a tool for assessing hand use and communicativeness in children with Rett Syndrome. Brain Dev 2005; 27: 95-6. http://dx.doi.org/10.1016/j.braindev.2005.03.019

[28] Yasuhara A, Sugiyama Y. Music therapy for children with Rett syndrome. Brain Dev 2001; 23: 82-4. http://dx.doi.org/10.1016/S0387-7604(01)00336-9

[29] Von Tetzchner S, Jacobsen KH, Smith L, Skjeldal $\mathrm{OH}$, Heiberg A, Fagan JF. Vision, cognition and developmental characteristics of girls and women with Rett syndrome. Develop Med Child Neurol 1996; 38: 212-25. http://dx.doi.org/10.1111/j.1469-8749.1996.tb15083.x

[30] Weaving LS, Ellaway CJ, Gécz J, Christodoulou J. Rett syndrome: clinical review and genetic update. J Med Genet 2005; 42: 1-7. http://dx.doi.org/10.1136/jmg.2004.027730

[31] Law M, Baptiste S, Mills J. Client-centred practice: what does it mean and does it make a difference?. Can J Occup Ther 1995; 62: 250-7. http://dx.doi.org/10.1177/000841749506200504

[32] Sliwa J. Intervention guidelines for the client-centered practice of occupational therapy. Am J Occup Ther 1988; 42: 403-404.

http://dx.doi.org/10.5014/ajot.42.6.403b

[33] Capio CM, Poolton JM, Sit CHP, Eguia KF, Masters RSW. Reduction of errors during practice facilitates fundamental movement skill learning in children with intellectual disabilities. J Intellectual Disabil Res 2013; 57: 295-305 http://dx.doi.org/10.1111/j.1365-2788.2012.01535.x

\section{DOI: http://dx.doi.org/10.6000/2292-2598.2016.04.03.1}

(C) 2016 Mraz et al.; Licensee Lifescience Global.

This is an open access article licensed under the terms of the Creative Commons Attribution Non-Commercial License (http://creativecommons.org/licenses/by-nc/3.0/) which permits unrestricted, non-commercial use, distribution and reproduction in any medium, provided the work is properly cited. 